\title{
All quantum states useful for teleportation are nonlocal resources
}

\author{
Daniel Cavalcanti, ${ }^{1}$ Antonio Acín, ${ }^{2,3}$ Nicolas Brunner, ${ }^{4,5}$ and Tamás Vértesi ${ }^{6}$ \\ ${ }^{1}$ Centre for Quantum Technologies, University of Singapore, 117542, Singapore \\ ${ }^{2}$ Institut de Ciencies Fotoniques, 08860 Castelldefels (Barcelona), Spain \\ ${ }^{3}$ Institució Catalana de Recerca i Estudis Avançats, Lluis Companys 23, 08010 Barcelona, Spain \\ ${ }^{4}$ H. H. Wills Physics Laboratory, University of Bristol, Tyndall Avenue, Bristol BS8 1TL, United Kingdom \\ ${ }^{5}$ Département de Physique Théorique, Université de Genève, 1211 Genève, Switzerland \\ ${ }^{6}$ Institute for Nuclear Research, Hungarian Academy of Sciences, P.O. Box 51, H-4001 Debrecen, Hungary
}

(Received 29 September 2012; published 8 April 2013)

\begin{abstract}
Understanding the relation between the different forms of inseparability in quantum mechanics is a longstanding problem in the foundations of quantum theory and has implications for quantum information processing. Here we make progress in this direction by establishing a direct link between quantum teleportation and Bell nonlocality. In particular, we show that all entangled states which are useful for teleportation are nonlocal resources, i.e., lead to deterministic violation of Bell's inequality. Our result also extends the phenomenon of superactivation of quantum nonlocality, recently proven by C. Palazuelos [Phys. Rev. Lett. 109, 190401 (2012)], and suggests that the latter might in fact be more general than initially thought.
\end{abstract}

DOI: 10.1103/PhysRevA.87.042104

PACS number(s): 03.65.Ud

\section{INTRODUCTION}

The fact that quantum mechanics is at odds with the principle of locality, although once viewed as evidence of the failure of the model, is now recognized as a fundamental aspect of quantum theory. The notion of inseparability in quantum mechanics appears under several forms. At the algebraic level is the concept of quantum entanglement. Entanglement is the resource for numerous quantum information protocols, in particular for quantum teleportation [1], which plays a central role in quantum communications and computation. The strongest notion of quantum inseparability is Bell nonlocality [2]. Distant observers sharing an entangled state can, by performing suitably chosen local measurements, generate correlations that cannot be reproduced by any local hiddenvariable model [3], as witnessed by the violation of a Bell inequality.

Understanding how these various forms of inseparability relate to each other is a long-standing problem, important from both fundamental and applied points of view. Although entanglement and nonlocality were first thought to be equivalent, it was shown that there exist mixed entangled states which are local, that is, admit a local hidden variable model [4], even for the most general type of local measurements [5]. It was then shown by Popescu [6] that some of these local entangled states are nevertheless useful for teleportation, which led him to argue that teleportation and Bell nonlocality are inequivalent forms of inseparability. Later, it was shown [7] that all two-qubit states violating the simplest Bell inequality, due to Clauser, Horne, Shimony, and Holt (CHSH), are useful for teleportation, reviving the hope for a link between teleportation and nonlocality.

Our main result is to show that a strong link between teleportation and nonlocality does indeed exist. In particular, we prove that all entangled states useful for teleportation are nonlocal resources, that is, can be used to violate a Bell inequality deterministically. The key point in our work is to allow the parties to use several copies of the state. More precisely, for all states $\rho$ useful for teleportation [8], there exists a finite number $k$ such that $k$ copies of $\rho$, i.e., $\rho^{\otimes k}$, deterministically violate a Bell inequality.

As a by-product, our results also provide examples of superactivation of quantum nonlocality. The fact that, by combining several copies of a state that admits a local model, it becomes possible to violate a Bell inequality is known as superactivation of quantum nonlocality. This represents an example of the phenomenon of activation in quantum mechanics, through which the judicious combination of several quantum entities becomes more powerful than the sum of the parts. Celebrated examples of activation were demonstrated in entanglement theory [9] and quantum channel theory [10]. In the case of nonlocality, some forms of activation in specific contexts were demonstrated, for instance, when postselection is considered [11-14], when several copies of the quantum state are distributed in a quantum network [15-18], when the number of measurements considered are restricted [19], when taking the amount of violation of a Bell inequality as a figure of merit [20], or in the case of general nonsignaling probability distributions [21]. However, the first example of superactivation of quantum nonlocality in the most natural scenario consisting of two parties who do not perform any local preprocessing of their quantum state is due to Palazuelos [22]. As shown below, our findings also allow us to extend Palazuelos's result and demonstrate additional examples of superactivation of quantum nonlocality in the bipartite and multipartite scenarios, suggesting that this phenomenon might be more general than initially thought.

\section{CONNECTING TELEPORTATION AND NONLOCALITY}

In what follows we say that a state $\rho$ is $k$-copy nonlocal if $\rho^{\otimes k}$ is nonlocal for some $k$. Given a state $\rho$, we focus on its entanglement fraction, defined by the maximum overlap of $\rho$ with a maximally entangled state. The main result of this paper is the following: Every quantum state acting on $C^{d} \otimes C^{d}$ with entanglement fraction higher than $1 / d$ is $k$-copy nonlocal.

It turns out that having an entanglement fraction higher than $1 / d$ is a necessary and sufficient condition for a state to 
provide a quantum advantage for teleportation [8]. Thus, our result implies that every state that is useful for teleportation is a nonlocal resource.

The proof of our result proceeds as follows: (i) we first show that every entangled isotropic state is $k$-copy nonlocal and then (ii) show that point (i) implies our main result.

In order to prove point (i) we essentially follow and slightly improve the proof by Palazuelos for the existence of superactivation of nonlocality [22]. This was based on the Khot-Visnoi Bell inequality [23,24],

$$
\beta_{K V}=\sum_{x, y=1}^{N} \sum_{a, b=1}^{n} c_{x, y, a, b} P(a b \mid x y) \leqslant \kappa_{\mathrm{loc}},
$$

where $\kappa_{\text {loc }}$ is the local bound and $c_{x, y, a, b}$ are positive coefficients. The following upper bound is known for $\kappa_{\text {loc }}$ :

$$
\kappa_{\mathrm{loc}} \leqslant C / n,
$$

where $N=2^{n} / n$ is the number of measurements per party, $n$ is the number of measurement outcomes [25], and $C$ is a universal constant. Moreover, it is known that there exist local measurements on a maximally entangled state of local dimension $n$ which produce a probability distribution such that

$$
\beta_{K V} \geqslant C^{\prime} /(\ln n)^{2}
$$

where $C^{\prime}$ is another universal constant. Since both the local bound and quantum violation depend on the dimension, it is useful to define the nonlocality fraction, given by

$$
\Gamma=\beta_{K V} / \kappa_{\text {loc }} \text {. }
$$

So we have a nonlocal probability distribution if $\Gamma>1$. In what follows we sometimes denote by $\Gamma(\rho)$ the nonlocal fraction of the probability distribution obtained by local measurements applied to state $\rho$.

Consider now the isotropic states, given by

$$
\rho_{\text {iso }}(F)=F\left|\Phi_{d}\right\rangle\left\langle\Phi_{d}\right|+(1-F) \frac{\mathbb{1}-\left|\Phi_{d}\right\rangle\left\langle\Phi_{d}\right|}{d^{2}-1},
$$

where $F=\left\langle\Phi_{d}\left|\rho_{\text {iso }}(d)\right| \Phi_{d}\right\rangle$ is the entanglement fraction [26] of the state $\rho_{\text {iso }}(F)$.

We can write $k$ copies of the isotropic state as

$$
\rho_{\text {iso }}^{\otimes k}=F^{k}\left|\Phi_{d^{k}}\right\rangle\left\langle\Phi_{d^{k}}\right|+\cdots+(1-F)^{k} \frac{\left(\mathbb{1}-\left|\Phi_{d}\right\rangle\left\langle\Phi_{d}\right|\right)^{\otimes k}}{\left(d^{2}-1\right)^{k}},
$$

where we have used the fact that the tensor product of $k$ maximally entangled states of local dimension $d$ is a maximally entangled state of local dimension $d^{k}$. One can use bounds (1) and (3) to show that the nonlocal fraction of $\rho_{\text {iso }}^{\otimes k}$ satisfies

$$
\begin{aligned}
\Gamma\left(\rho_{\text {iso }}^{\otimes k}\right)= & F^{k} \Gamma\left(\left|\Phi_{d^{k}}\right\rangle\left\langle\Phi_{d^{k}}\right|\right)+\cdots \\
& +(1-F)^{k} \Gamma\left(\frac{\left(\mathbb{1}-\left|\Phi_{d}\right\rangle\left\langle\Phi_{d}\right|\right)^{\otimes k}}{\left(d^{2}-1\right)^{k}}\right) \\
\geqslant & F^{k} \Gamma\left(\left|\Phi_{d^{k}}\right\rangle\left\langle\Phi_{d^{k}}\right|\right) \\
\geqslant & \frac{C^{\prime}}{C} F^{k} \frac{d^{k}}{(k \ln d)^{2}} .
\end{aligned}
$$

Thus, in the case $F>1 / d$ the right-hand side of (7) increases with $k$. This implies that there will be a given number of copies $k^{\prime}$ for which $\Gamma\left(\rho_{\text {iso }}^{\otimes k^{\prime}}\right)>1$, which implies that $\rho_{\text {iso }}^{\otimes k^{\prime}}$ is nonlocal. Since $F=1 / d$ is the separability bound for the isotropic state, we conclude point (i), i.e., that every entangled isotropic state is $k$-copy nonlocal.

We now proceed to show our main result. In order to see this, first notice that any quantum state $\rho_{0}$ can be depolarized into an isotropic state by the application of randomly chosen unitaries as

$$
\rho_{\mathrm{iso}}\left(F_{0}\right)=\sum_{i} p_{i}\left(U_{i} \otimes U_{i}^{*}\right) \rho_{0}\left(U_{i} \otimes U_{i}^{*}\right)^{-1}
$$

while keeping the entanglement fraction $F_{0}$, i.e., $F_{0}=$ $\max _{\Psi}\left\langle\Psi\left|\rho_{0}\right| \Psi\right\rangle$, where the maximum is taken over all $d \times d$ maximally entangled states $\Psi[26,27]$. Since the initial state has an entanglement fraction higher than $1 / d$, the resulting isotropic state is entangled and, as we have just shown, $k$-copy nonlocal. That is, the state

$$
\rho_{\text {iso }}\left(F_{0}\right)^{\otimes k}=\sum_{i_{1}, \ldots, i_{k}} p_{i_{1}} \cdots p_{i_{k}} \mathcal{U}_{i_{1}, \ldots, i_{k}} \rho_{0}^{\otimes k} \mathcal{U}_{i_{1}, \ldots, i_{k}}^{-1},
$$

where $\mathcal{U}_{i_{1} \ldots i_{k}}=\left(U_{i_{1}} \otimes \cdots \otimes U_{i_{k}}\right)_{\text {Alice }} \otimes\left(U_{i_{1}}^{*} \otimes \cdots \otimes U_{i_{k}}^{*}\right)_{\mathrm{Bob}}$, violates the Khot-Visnoi Bell inequality. This means that there is a Bell operator $\mathcal{B}$ such that $\operatorname{tr}\left[\mathcal{B} \rho_{\text {iso }}\left(F_{0}\right)^{\otimes k}\right]>L(L$ is the local bound of the corresponding Bell inequality). But then there is (at least) one term in the sum of (9) such that $\operatorname{tr}\left(\mathcal{B} \mathcal{U}_{i_{1}, \ldots, i_{k}} \rho_{0}^{\otimes k} \mathcal{U}_{i_{1}, \ldots, i_{k}}^{-1}\right)>L$. Since the local unitaries can be absorbed into the choice of local measurements, it follows that the original state $\rho_{0}^{\otimes k}$ is also $k$-copy nonlocal. This ends the proof of our main result.

\section{SUPERACTIVATION OF NONLOCALITY}

In Ref. [22], Palazuelos studied the nonlocal properties of many copies of the isotropic state (5). It is known that there exist ranges of the entanglement fraction $F$, given by $F \leqslant F_{\text {loc }}$, such that this state is local; i.e., the probability distributions of the results of local measurements applied to (5) can be explained by a (classical) local model [28]. However, Palazuelos showed that sufficiently many copies of a local isotropic state, i.e., $\rho_{\text {iso }}\left(F_{L}\right)^{\otimes k}$, can violate a Bell inequality if $d \geqslant 8$ [22]. This shows that quantum nonlocality can be superactivated.

Point (i) extends the result by Palazuelos in two senses. First, it shows superactivation of nonlocality for the whole range of the parameter $F$ for which the isotropic state is entangled and has a local model (see Fig. 1). Second, it is valid for any dimension, while the minimum local dimension used in the proof of Ref. [22] is $d=8$.

Furthermore our main result allows us to construct additional examples of superactivation of nonlocality. For instance, consider mixtures of an arbitrary pure state and the maximally mixed noise,

$$
\sigma=p|\psi\rangle\langle\psi|+(1-p) \frac{\mathbb{1}}{d^{2}} .
$$

An arbitrary bipartite pure state $|\psi\rangle$ reads, in its Schmidt decomposition, $|\psi\rangle=\sum_{i=1}^{d} \lambda_{i}|i i\rangle$. Its entanglement fraction can be written as

$$
F_{\psi}=\left|\left\langle\Phi_{d} \mid \psi\right\rangle\right|^{2}=\frac{\left(\sum_{i=1}^{d} \lambda_{i}\right)^{2}}{d} .
$$




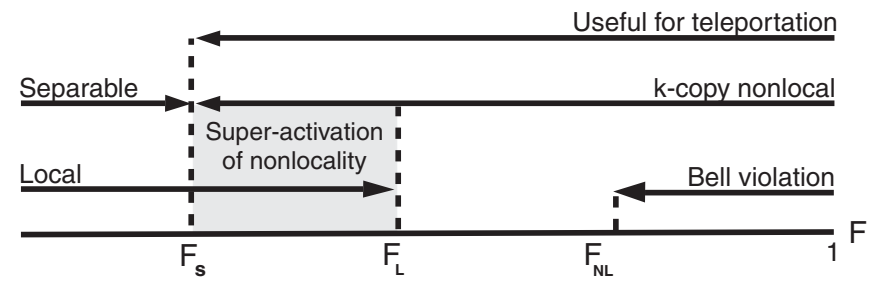

FIG. 1. Nonlocal properties of the isotropic state (5). This state is separable for $F \leqslant F_{s}$, has a local model for $F \leqslant F_{L}$ [28], and is known to violate a Bell inequality for $F_{L}<F_{N L}<F$ [29]. Here we show that several copies of the isotropic state are nonlocal if it is entangled, that is, if $F_{s}<F$. The state is useful for teleportation down to the separability limit $F_{s}$.

By our main result, $\sigma$ is $k$-copy nonlocal if $F_{\sigma}=\left\langle\Phi_{d}|\sigma| \Phi_{d}\right\rangle=$ $p F_{\phi}+(1-p) / d^{2}>1 / d$, which translates into

$$
p>\frac{d-1}{d\left(\sum_{i=1}^{d} \lambda_{i}\right)^{2}-1} .
$$

It was shown in Ref. [28] that there exists a range of the noise parameter $p$ for which state (10) is known to be entangled and local [28]. Comparing the bounds in [28] with (12), it is possible to identify additional examples of superactivation of quantum nonlocality. In particular, for $d=2$ the value of (12) gives exactly the separability bound for state $\sigma$.

Another class of states with a local model is given by [28]

$$
\rho=p|\psi\rangle\langle\psi|+(1-p) \rho_{A} \otimes \frac{\mathbb{1}}{2},
$$

where $|\psi\rangle=\cos \theta|00\rangle+\sin \theta|11\rangle(0 \leqslant \theta \leqslant \pi / 4)$ is an arbitrary two-qubit state with reduced state $\rho_{A}$. This state has a local model for $p \leqslant 5 / 12$ [28]. Imposing that the entanglement fraction of (13) is bigger than $1 / 2$ implies $p>1 /[1+2 \sin (2 \theta)]$. Using the partial transpose criterion, the separability limit is found to be $p_{S}=1 / 3$. Hence state (13) for all $0<\theta \leqslant \pi / 4$ when $p>1 /[1+2 \sin (2 \theta)]$ is $k$-copy nonlocal, and there is superactivation (considering the local limit $\left.p_{L}=5 / 12\right)$ above $\theta>(1 / 2) \arcsin (7 / 10) \sim 0.3877 \mathrm{rad}$.

Our result can also be used to show superactivation of nonlocality in the multipartite scenario. In Ref. [30] a genuinely entangled multipartite state of three qubits with a local model was presented. It turns out that the reduced states of this tripartite state are entangled two-qubit isotropic states. Thus, bipartite nonlocality can be obtained by $k$ copies of this tripartite state.

\section{DISCUSSION}

We have shown that all entangled states useful for teleportation are nonlocal resources, in the sense that a Bell inequality is always violated deterministically when suitable local measurements are performed on a sufficiently large number of copies of the state. Thus, our result establishes a direct and general link between teleportation and nonlocality, two central forms of inseparability in quantum mechanics previously thought to be unrelated [6]. Our results also suggest that the superactivation of quantum nonlocality might be more general than expected, as any entangled state useful for teleportation that has a local model provides an instance of this phenomenon. In fact, we use this connection to provide explicit examples of superactivation.

Our paper raises several questions. First, it would be interesting to see if the converse of our main result holds, that is, if any state that violates a Bell inequality is useful for teleportation. Indeed, such a link has been established for the particular case of the CHSH Bell inequality [7]. If this is the case, then teleportation and nonlocality would turn out to be equivalent. Moreover, Bell inequality violation would represent a device-independent test for usefulness in teleportation.

Second, we have shown that a large class of entangled states display nonlocal correlations, possibly after taking several copies of it. Thus it is natural to ask whether this result applies to any entangled state. A positive answer to this question would demonstrate a direct link between entanglement and nonlocality, which would considerably improve our understanding of these concepts.

We believe, however, that answering this question is highly challenging. Nevertheless, partial answers would already represent breakthroughs. For instance, is it the case that any entangled state that is distillable is also $k$-copy nonlocal? Note that our result applies to states which are distillable, as an entanglement fraction higher than $1 / d$ is a sufficient condition for entanglement distillability. This condition is, however, not necessary, and therefore there exist distillable states to which our result does not apply. More ambitious is the case of bound entangled states, i.e., entangled states that cannot be distilled. If such states were shown to violate a Bell inequality, it would disprove a long-standing conjecture made by Peres [31]. More generally, this would show that bipartite nonlocality does not imply entanglement distillability, a result known to hold true in the multipartite setting [32].

\section{ACKNOWLEDGMENTS}

We thank Y.-C. Liang and C. Palazuelos for useful comments. This work was supported by the National Research Foundation and the Ministry of Education of Singapore, the UK EPSRC, Spanish Project No. FIS201014830 and Generalitat de Catalunya, the European PERCENT ERC Starting Grant, Q-Essence, QCS and DIQIP projects, the Hungarian National Research Fund OTKA (Grant No. PD101461), a János Bolyai Grant of the Hungarian Academy of Sciences and the TÁMOP-4.2.2.C-11/1/KONV-2012-0001 project, and the Swiss National Science Foundation (Grant No. PP00P2_138917). D.C. and N.B. thank A.A. for the hospitality at ICFO. D.C. thanks N.B. for the hospitality at the University of Bristol.
[1] C. H. Bennett, G. Brassard, C. Crepeau, R. Jozsa, A. Peres, and W. K. Wootters, Phys. Rev. Lett. 70, 1895 (1993).

[2] J. S. Bell, Physics 1, 195 (1964).
[3] A. Einstein, B. Podolsky, and N. Rosen, Phys. Rev. 47, 777 (1935).

[4] R. F. Werner, Phys. Rev. A 40, 4277 (1989). 
[5] J. Barrett, Phys. Rev. A 65, 042302 (2002).

[6] S. Popescu, Phys. Rev. Lett. 72, 797 (1994).

[7] R. Horodecki, M. Horodecki, and P. Horodecki, Phys. Lett. A 222, 21 (1996).

[8] M. Horodecki, P. Horodecki, and R. Horodecki, Phys. Rev. A 60, 1888 (1999).

[9] P. Horodecki, M. Horodecki, and R. Horodecki, Phys. Rev. Lett. 82, 1056 (1999).

[10] G. Smith and J. Yard, Science 321, 1812 (2008).

[11] S. Popescu, Phys. Rev. Lett. 74, 2619 (1995).

[12] A. Peres, Phys. Rev. A 54, 2685 (1996).

[13] N. Gisin, Phys. Lett. A 210, 151 (1996).

[14] Ll. Masanes, Y.-C. Liang, and A. C. Doherty, Phys. Rev. Lett. 100, 090403 (2008).

[15] A. Sen(De), U. Sen, C. Brukner, V. Bužek, and M. Żukowski, Phys. Rev. A 72, 042310 (2005).

[16] D. Cavalcanti, M. L. Almeida, V. Scarani, and A. Acín, Nat. Commun. 2, 184 (2011).

[17] D. Cavalcanti, R. Rabelo, and V. Scarani, Phys. Rev. Lett. 108, 040402 (2012).

[18] A. Wójcik, J. Modlawska, A. Grudka, and M. Czechlewski, Phys. Lett. A 374, 4831 (2010); W. Kłobus, W. Laskowski, M. Markiewicz, and A. Grudka, Phys. Rev. A 86, 020302(R) (2012).

[19] M. Navascués and T. Vértesi, Phys. Rev. Lett. 106, 060403 (2011).
[20] Y.-C. Liang and A. C. Doherty, Phys. Rev. A 73, 052116 (2006).

[21] N. Brunner, D. Cavalcanti, A. Salles, and P. Skrzypczyk, Phys. Rev. Lett. 106, 020402 (2011).

[22] C. Palazuelos, Phys. Rev. Lett. 109, 190401 (2012).

[23] S. A. Khot and N. K. Vishnoi, in Proceedings 46th FOCS, Pittsburgh, 2005 (IEEE, Piscataway, NJ, 2005), pp. 53-62.

[24] H. Buhrman, O. Regev, G. Scarpa, and R. de Wolf, in 26th IEEE Conference on Computational Complexity (CCC 11), San Diego, 2011 (IEEE, Piscataway, NJ, 2011), pp. 157166.

[25] The Khot-Visnoi game was initially defined for $n$ being a power of 2. However, it was generalized for general $n$ in C. Palazuelos, arXiv:1206.3695.

[26] M. Horodecki and P. Horodecki, Phys. Rev. A 59, 4206 (1999).

[27] D. Gross, K. Audenaert, and J. Eisert, J. Math. Phys. 48, 052104 (2007).

[28] M. L. Almeida, S. Pironio, J. Barrett, G. Tóth, and A. Acín, Phys. Rev. Lett. 99, 040403 (2007).

[29] D. Collins, N. Gisin, N. Linden, S. Massar, and S. Popescu, Phys. Rev. Lett. 88, 040404 (2002).

[30] G. Tóth and A. Acín, Phys. Rev. A 74, 030306(R) (2006).

[31] A. Peres, Found. Phys. 29, 589 (1999).

[32] T. Vértesi and N. Brunner, Phys. Rev. Lett. 108, 030403 (2012). 